\title{
Large scale magnetic fields in discs: jets and reconnection $\mathrm{X}$-winds
}

\author{
Jonathan Ferreira, Nicolas Bessolaz, Claudio Zanni, \\ and Céline Combet
}

Laboratoire d'Astrophysique de Grenoble, F-38041 Grenoble, France

email: Jonathan.Ferreira@obs.ujf-grenoble.fr

\begin{abstract}
In this contribution we first briefly review our current knowledge on the physics of accretion discs driving self-confined jets. It will be shown that a large scale magnetic field is expected to thread the innermost disc regions, giving rise to a transition from an outer standard accretion disc to an inner jet emitting disc. We then report new progresses on the theory of stardisc interaction, allowing to explain the formation of accretion funnel flows with stellar dipole fields consistent with observational constraints. Such a connection is now not only probed by modern observations but it is also requested for spinning down protostars, which are known to be both actively accreting and contracting. This spin down most probably relies on the angular momentum removal by ejection. Two such scenarios will be addressed here, namely "accretionpowered stellar winds" (Matt \& Pudritz 2005) and "Reconnection X-winds" (Ferreira, Pelletier $\&$ Appl 2000). The latter can slow down a protostar on time scales shorter or comparable to the embedded phase. It will be shown that these two scenarios are not incompatible and that transitions from one to another may even occur as they mainly depend on the stellar dynamo.
\end{abstract}

Keywords. Accretion discs, magnetohydrodynamics, stars: pre-main sequence, stars: magnetic fields, stars: rotation, ISM: jets and outflows.

\section{Introduction}

Actively accreting classical T Tauri stars (CTTS) often display supersonic collimated jets on scales of a few 10-100 AU in low excitation optical forbidden lines. Molecular outflows observed in younger Class 0 and I sources may be powered by an inner unobserved optical jet. These jet signatures are correlated with the infrared excess and accretion rate of the circumstellar disc (Cabrit et al. 1990; Hartigan et al. 1995). It is therefore widely believed that the accretion process is essential to the production of jets. For quite a while, the precise physical connection remained a matter of debate: do the jets emanate from the star, the circumstellar disc or the magnetospheric star-disc interaction? This issue seems now to be almost settled: while all these wind components are probably present, magnetized disc winds would be responsible for most of the mass loss (see Ferreira et al. 2006a and S. Cabrit's contribution).

The basic and universal accretion-ejection mechanism would then be the following: an accretion disc around a central object can - under certain conditions and whatever the nature of this object (star or compact object) - drive jets through the action of large scale magnetic fields. These fields would tap the mechanical energy released by mass accretion within the disc and transfer it to an ejected fraction. The smaller the fraction, the larger the final jet velocity. One thing that must be understood is how the presence of these jets modifies the nature of the underlying accretion flow. Many papers in the literature actually assume that the accretion disc resembles a standard accretion disc (hereafter SAD), as first described by Shakura \& Sunyaev (1973). Thus, although 
it was soon recognized that ejection and accretion were tightly related (Blandford \& Payne 1982; Konigl 1989), a truly self-consistent model appeared only lately (Ferreira \& Pelletier 1995; Ferreira 1997; Casse \& Ferreira 2000; Ferreira \& Casse 2004). To date, this is the only published magneto-hydrodynamic (MHD) model that describes in a selfconsistent way the physics of an accretion disc threaded by a large scale magnetic field and giving rise to self-collimated jets. We term such a disc a Jet Emitting Disc, hereafter a JED. This model is unique in the sense that it provides both the physical conditions within the disc required to steadily launch jets and the distributions of all quantities in space (although the self-similar assumption used introduces some unavoidable biases). Most of its results have been confirmed by numerical MHD simulations using either the VAC code (Casse \& Keppens 2002, 2004) or the FLASH code (Zanni et al. 2007).

In this contribution, we present some results on how the presence of a large scale vertical magnetic field deeply alters the disc dynamics (Section 2). Section 3 addresses new results on the star-disc interaction obtained with two MHD codes, VAC and PLUTO. It will be argued that such an interaction probably leads to a systematic spin up of the protostar. Section 4 is then devoted to the only model so far that allows a magnetic brake down of a protostar during its embedded phase.

\section{Magnetic fields in accretion discs}

The necessary condition for launching a self-collimated jet from a Keplerian accretion disc is the presence of a large scale vertical magnetic field close to equipartition (Ferreira \& Pelletier 1995), namely

$$
B_{z} \simeq 0.2\left(\frac{M}{M_{\odot}}\right)^{1 / 4}\left(\frac{\dot{M}_{a}}{10^{-7} M_{\odot} / y r}\right)^{1 / 2}\left(\frac{r}{1 \mathrm{AU}}\right)^{-5 / 4+\xi / 2} \mathrm{G},
$$

where $\xi$ is the disc ejection efficiency as measured by a varying disc accretion rate, namely $\dot{M}_{a} \propto r^{\xi}$. The value of this magnetic field is far smaller than the one estimated from the interstellar magnetic field assuming either ideal MHD or $B \propto n^{1 / 2}$ (Heiles et al. 1993; Basu \& Mouschovias 1994). This implies some decoupling between the infalling/accreting material and the magnetic field in order to get rid off this field. This issue is still under debate. The question is therefore whether accretion discs can build up their own large scale magnetic field (dynamo) or if they can drag in the interstellar magnetic field? Although no large scale fields have been provided by a self-consistent disc dynamo, this scenario cannot be excluded. However the latter scenario (advection) seems a bit more natural (see F. Shu's contribution).

A picture, that can be applied to accretion discs around both young stars and compact objects, is now gradually emerging. A large scale magnetic field is thought to be dragged in by the accretion flow and concentrated in the innermost disc regions. This is consistent with the SAD theory. As a result of the interplay between advection (due to accretion) and turbulent diffusion, the large scale magnetic field scales as $B_{z} \propto r^{-\mathcal{R}_{m}}$, where $\mathcal{R}_{m}$ of order unity is the effective magnetic Reynolds number. As a consequence, one gets a disc magnetization $\mu=B_{z}^{2} / \mu_{o} P$ where $P$ is the gas pressure which naturally increases towards the inner regions (Ferreira et al. 2006b). Such a field triggers the magneto-rotational instability (Balbus 2003) in the outer disc regions, producing thereby a standard accretion disc with no ejection (note that a thermally driven or photo-evaporated disc wind is of course clearly possible). When the field reaches equipartition, the accretion flow switches from a SAD to a JED, the latter driving self-confined jets. The physics of this inner disc is thus no longer governed by the radial transport of angular momentum. 
This picture puts a strong emphasis on the strength of the disc large scale magnetic field $\left(B_{z}\right)$. Probing inner disc regions where $\mu \sim 1$ (fields given by Eq. 1) may appear too demanding as magnetic fields are very difficult to measure. However, Donati et al. (2005) did this tour de force using the spectro-polarimeter ESPadOnS and found a $\sim \mathrm{kG}$ field at $0.05 \mathrm{AU}$ around FU Ori (a field actually larger than equipartition!). Moreover, $\mathrm{T}$ Tauri discs may not all reach equipartition fields if these mostly depend on interstellar field advection. This is indeed suggested by the striking results obtained by Ménard \& Duchêne (2004). Using a sample of CTTS, these authors found that CTTS are oriented randomly with respect to the local interstellar field. However, sources (discs) with strong outflows are mostly perpendicular to the field (i.e. jets are aligned to it, Strom et al. 1986), whereas sources with no jet detection are parallel. That could be a hint that, only in the former case, field dragging leads to the presence of inner JEDs.

An indirect way to probe this region is to look at jet kinematic properties and in particular at the rotation. Current observations clearly favor self-confined jets launched from some radial extension in the disc (say from 0.1 to 0.5-2 AUs, see Ferreira et al. 2006a for more details). However, cold models with a low ejection efficiency $\xi \sim 0.01$ hence a magnetic lever arm parameter $\lambda=1+1 / 2 \xi \sim 50$ are excluded. Only "warm" solutions with $\xi \sim 0.1(\lambda \sim 10)$ are fully compatible with current observations (mass flux, velocities, collimation). Such models require heat input at the upper disc surface layers in order to allow more mass to be loaded onto the field lines. The origin of this heat deposition remains an open question. It cannot be solely due to illumination by stellar UV and X-ray radiation (Garcia et al. to be submitted). Alternatively, the turbulent processes responsible for the required magnetic diffusivity inside the disc might also give rise to a turbulent vertical heat flux, leading to dissipation at the disc surface layers. It is interesting to note that, in current MHD simulations of the magneto-rotational instability, a magnetically active "corona" is quickly established (Stone et al. 1996; Miller \& Stone 2000). Although no 3D simulation has been run with open magnetic field lines, this result is rather promising. Indeed, it might be an intrinsic property of the MHD turbulence in accretion discs, regardless of the launching of jets (see also arguments developed by Kwan 1997 and Glassgold et al. 2004).

From the observational point of view, JED and SAD emission properties are quite different. While in a SAD all the released accretion power is radiated away at the disc surfaces, in a JED this power is feeding the two jets. As a consequence, only a small fraction (of order $h / r$ ) of the available power is put into the disc luminosity (Ferreira \& Pelletier 1995). This translates right away into a lack of disc emission from the innermost ejecting parts: the spectral energy distribution would thus appear flatter than the usual $-4 / 3$ scaling. But more interestingly, the disc is much less dense than a corresponding SAD at the same radius (with the same $\dot{M}_{a}$, Combet \& Ferreira, submitted). This is a straightforward consequence of a much larger accretion velocity due to the dominant jet torque. This could lead to optically thin parts in the JED but, in any case, to a sharp decrease of both the disc surface density $\Sigma(r)$ and scale height $h(r)$ at the SADJED transition radius $r_{J}$. This radius is unknown as it depends on the magnetic flux $\Phi$ available in the disc (and may vary from one object to another as discussed above), but may lead to observational investigations.

This last property is very interesting since Masset et al. (2006) have shown that a transition of this kind would act as a trap for low mass protoplanetary embryo $(M<$ $\left.15 M_{\oplus}\right)$. Indeed, Type I inwards migration is due to the differential Lindblad torque arising from the planetesimal interaction with the viscous disc. But this negative torque is strongly reduced at the transition radius and balanced by the positive corotation torque (which is due to the exchange of angular momentum between the planetesimal 
and trapped disc material in its vicinity). Thus, these planetesimals would be halted at $r_{J}$ which may be as large as $1 \mathrm{AU}$, long before the disc truncation radius due to the star-disc interaction.

\section{The star-disc interaction}

Once they become visible in the optical, $\mathrm{T}$ Tauri stars exhibit rotational periods of the order of 10 days, which is much smaller than expected (Bouvier et al. 1997; Rebull et al. 2002). This implies a very efficient mechanism of angular momentum removal from the star during its embedded phase. Moreover, a T Tauri star seems to evolve with an almost constant rotational period although it undergoes some contraction and is still actively accreting disc material for roughly a million years. This is a major issue in star formation, unsolved yet, but one solution to this paradox is the star-disc interaction.

\subsection{The Gosh \& Lamb picture}

Angular resolution is not yet sufficient to directly image this region (of size $0.1 \mathrm{AU}$ or less: it would require optical interferometry) but there have been mounting spectroscopic and photometric evidences that the disc is truncated by a stellar magnetosphere (assumed to be a dipole) and that accretion proceeds along magnetic funnels or curtains towards the magnetic poles (see Bouvier et al. 2007 and references therein). This gave rise to the so-called disc locking paradigm, where it is assumed that the stellar angular momentum could be transferred to the disc (Ghosh et al. 1977; Collier Cameron \& Campbell 1993; Armitage \& Clarke 1996). Unfortunately, this idealized picture can probably not be maintained (see a thorough discussion in Matt \& Pudritz 2005b).

The simple reason is that accretion onto the star and this "strict" disc locking mechanism are two contradictory requirements. Let $\Omega_{*}$ be the angular velocity of the star. Its magnetosphere will try to make the disc material corotate with the protostar so that the sign of the torque depends directly on the relative angular velocity. Stellar magnetic field lines threading the disc beyond the rotation radius $r_{c o}=\left(G M / \Omega_{*}^{2}\right)^{1 / 3}$ exert a positive torque, whereas they brake down the disc material below $r_{c o}$. Let us also define the truncation radius $r_{t}$ below which the stellar magnetic field is strong enough to "truncate" the disc by enforcing the material to flow along the field lines and no longer on the plane of the disc. Now, one can safely realize that accretion onto the star can only proceed if $r_{t}<r_{c o}$. In this situation, the stellar magnetic field can brake down both the disc and the material accreting in the funnel flows. This implies of course a stellar spin up by the disc material located below $r_{c o}$. The disc locking paradigm assumes that stellar field lines remain anchored beyond $r_{c o}$, giving hopefully rise to some angular momentum balance. But within this paradigm, the disc viscosity must be efficient enough so as to radially transport outwards both the disc and stellar angular momentum! This is unrealistic because the stellar angular momentum is far too large. Moreover, all numerical simulations done so far showed a fast opening of the field lines beyond $r_{c o}$ (through numerical reconnection), severing the causal link and thereby dramatically reducing this negative torque (Lovelace et al. 1995, 1999; Long et al. 2005). Although this effect is strongly dependent on the disc magnetic turbulent diffusivity, the main result is to spin up the star whenever $r_{t}<r_{c o}$ (Zanni et al, in prep).

Therefore, an important question is what determines the disc truncation radius $r_{t}$ ? In fact, two constraints must be simultaneously fulfilled for driving steady-state accretion funnel flows (Bessolaz et al, submitted). First, the poloidal stellar magnetic field must be strong enough to halt the accretion motion, namely $B_{z}^{2} / \mu_{o} \sim \rho u_{r}^{2}$. Second, the disc thermal pressure must be able to lift material vertically in order to initiate the accretion 
funnel flow, $B_{z}^{2} / \mu_{o} \sim P$. This last constraint is equivalent to an equipartition field, as first proposed by Pringle \& Rees (1972). Putting this two constraints together one derives a ratio of the disc truncation radius to the co-rotation radius

$$
\frac{r_{t}}{r_{c o}} \simeq 0.66 B_{*}^{4 / 7} \dot{M}_{a}^{-2 / 7} M_{*}^{-10 / 21} R_{*}^{12 / 7} P_{*}^{-2 / 3}
$$

where the disc accretion rate $\dot{M}_{a}$ has been normalized to $10^{-8} \mathrm{M}_{\odot} \mathrm{yr}^{-1}$, stellar dipole field $B_{*}$ to $150 \mathrm{G}$, mass to $0.5 \mathrm{M}_{\odot}$, radius to $3 \mathrm{R}_{\odot}$ and period $P_{*}$ to 8 days. These analytical constraints and estimates have been confirmed using MHD axisymmetric numerical simulations of a star-disc dipole interaction (Bessolaz et al. - VAC, Zanni et al. PLUTO, submitted). This is therefore a robust result. It shows that truncating discs can be done with a dipole field of several hundreds of Gauss (not kG !), consistent with both observations of magnetic fields (Donati, private communication) and sizes of inner disc holes (Najita et al. 2007). It confirms that for T Tauri parameters $r_{t}<r_{c o}$ and that the "disc locking" picture a la Ghosh \& Lamb is most probably not working.

\subsection{Stellar spin down via winds}

The obvious way to conciliate this result with the observational constraint that accreting stars are actually being spun down is via winds that would not exist without the presence of accretion. This has led Matt \& Pudritz (2005a) to propose the name of "accretion powered stellar winds".

Accretion onto the star takes place along closed magnetospheric field lines, shocks the stellar surface and releases there most of its mechanical energy (mostly UV emission). The idea is then that a fraction of this accretion-heated mass diffuses towards the magnetic pole until it reaches open field lines. A warm stellar wind can then be initiated. The problem with $\mathrm{T}$ Tauri stars is that they are rotating at about $10 \%$ of their break-up speed. This translates into a totally negligible magnetic acceleration (the stellar material is far too deep in the gravitational potential well). One has therefore to rely on pressuredriven winds (see eg. discussion in Ferreira et al. 2006a for "enhanced stellar winds"). Now, if that initial pressure is only thermal, then temperatures of several million degrees are required. This raises the critical issue of too strong emission losses due to this inner hot wind (see S. Matt's contribution). The alternative is to rely on a turbulent Alfvén wave pressure and would be less dissipative (Hartmann \& MacGregor 1980; DeCampli 1981). Note that the presence of turbulent MHD waves is expected in this context.

It should be noted that current MHD numerical simulations of star-disc interaction (e.g. Long et al. 2005; Zanni et al. 2007) do show a magnetic braking due to the opened stellar field lines. This has been interpreted as a "magnetic tower" since no real stellar wind was incorporated in the simulations. This is obviously a very promising issue. A thorough investigation should therefore be conducted in order to assess whether or not accretion-powered stellar winds of this kind can indeed (i) be dense enough and with a magnetic lever arm large enough to brake down the protostar, (ii) have radiative losses consistent with observations and (iii) do not pose any energetic problem like e.g. requiring to tap more than $50 \%$ of the accretion luminosity.

\section{Reconnection $\mathrm{X}$-winds}

Accretion-powered stellar winds are somehow designed to explain the "disc locking" paradigm for $\mathrm{T}$ Tauri stars, namely to maintain their low rotation rate despite accretion. But how can we explain that $\mathrm{T}$ Tauri stars do already rotate at $10 \%$ of their breakup speed? Numerical simulations of the collapse of rotating magnetized clouds succeed 
nowadays in explaining the formation of protostellar cores at break-up speeds thanks to magnetic braking (Banerjee \& Pudritz 2006; Machida et al. 2006). However, it is doubtful that such a braking could provide much lower initial rotation rates. One must then rely on some interaction between the protostar and its disc during the embedded phase (Class 0 and possibly Class I).

To our knowledge, the only model that addresses this issue is the Reconnection $\mathrm{X}$-wind model (Ferreira et al. 2000). In this model, it is assumed that the interstellar magnetic field is advected with the infalling material in such a way that a significant magnetic flux $\Phi$ is now threading the protostellar core and the inner disc regions (as simulations show). This self-gravitating core will develop a dynamo of some kind but whose outcome is assumed to be the generation of a dipole field with a magnetic moment parallel to the disc magnetic field. This is clearly an assumption as there is no theory of such a constrained dynamo that takes into account both the presence of an initial strong fossil field and the outer disc (see however Moss 2004). The coexistence of this dipolar stellar field with the outer disc field generates an X-type magnetic neutral line, where both fields cancel each other at a radius $r_{X}$. Note that such a magnetic configuration has been previously considered by Uchida \& Low (1981) and Hirose et al. (1997), but without taking into account the stellar rotation.

Let us assume that at $t=0$ the dipole is emerging from a protostar rotating at break-up speed so that $r_{X}=R_{*, 0}$ with $R_{*, 0}=R_{*}(t=0), M_{*, 0}=M_{*}(t=0), \dot{M}_{a, 0}=\dot{M}_{a}(t=0)$, $\Omega_{*, 0}=\sqrt{G M_{*, 0} / R_{*, 0}^{3}}$ and $B_{*, 0}=B_{*}(t=0)$. It is further assumed that the field threading the disc is strong enough to drive self-confined disc winds at these early stages. Then Eq. (2.1) applies and provides us the value of the required stellar field. What will be the consequences of this initial state?

From the point of view of the disc, nothing is changed beyond $r_{X}$ : a disc wind is taking place in the JED and disc material accretes by loosing its angular momentum in the jets. At $r_{X}$ however, magnetic reconnection converts closed stellar field lines and open disc field lines into open stellar field lines. Accreting material that was already at the disc surface at $r_{X}$ is now loaded into these newly opened field lines (there is a strong upward Lorentz force above $r_{X}$ ). Since these lines are now rotating at the stellar rotation rate, they exert a strong azimuthal force that drives ejection. This new type of wind has been called "Reconnection X-winds". Although material is ejected along field lines anchored onto the star, this is not a stellar wind since material did not reach the stellar surface and thus did not loose its rotational energy: it is much easier to accelerate matter under these circumstances.

Reconnection $\mathrm{X}$-winds are fed with disc material and powered by the stellar rotational energy. As a consequence, they exert a negative torque on the protostar which leads to a stellar spin down. On the other hand, an increase of the stellar angular velocity $\Omega_{*}$ is expected from both accretion and contraction, with a typical Kelvin-Helmoltz time scale of several $10^{5}$ yrs. Because of the huge stellar inertia, the evolution of $\Omega_{*}$ with time must be followed on these long time scales. One assumption used to compute the angular momentum history of the protostar on those scales is that $r_{X} \simeq r_{c o}$. Such an assumption relies on the possibility for the protostellar magnetosphere to evacuate angular momentum through violent ejection events (Reconnection X-winds) whenever $r_{X}>r_{c o}$, while quasi steady accretion columns form when $r_{X}<r_{c o}$. Consistently with $r_{X} \simeq r_{c o}$, a constant fraction $f=\dot{M}_{X} / \dot{M}_{a}$ is assumed on these long time scales, where $\dot{M}_{X}$ is the ejected mass flux in Reconnection X-winds, as well as a constant magnetic lever arm parameter $\lambda$. These winds are therefore best seen as violent outbursts carrying disc material (blobs?) and stellar angular momentum from the star-disc interaction, channeled 
and confined by the outer disc wind. Note that a conventional stellar wind would of course take place and fill in the inner field lines with mass, but its effect on the stellar spin evolution has been neglected in this work.

The global picture is then the following. As the protostar is being spun down, the corotation radius $r_{c o}$ increases and so must $r_{X}$. The stellar dipole field is assumed to follow $B_{\text {star }}=B_{*}\left(r / R_{*}\right)^{-n}$ where the index $n$ describes a deviation from a pure dipole in vacuum. Now, $r_{X}$ is defined by the cancellation of the stellar and disc field, whose scaling is very different from the former (see Eq. 2.1). The only way to ensure $r_{X} \simeq r_{c o}$ on these long time scales is then to decrease $\dot{M}_{a}$ in time as well. Note that this is not a surprise as the accretion rate onto the star is controlled by the star-disc interaction. Thus, while computing the stellar spin evolution in time $\Omega_{*}(t)$, starting from conditions prevailing in Class 0 objects and using $f, \lambda$ and $n$ as free parameters, one gets also $R_{*}(t), M_{*}(t)$ and $\dot{M}_{a}(t)$. Note that this global process of angular momentum removal is intimately related to the magnetic history of the protostar-disc system. Two additional ingredients are thus necessary: the amount of magnetic flux $\Phi$ threading the disc and how the stellar field $B_{*}$ evolves with time (through dynamo). The calculations reported in Ferreira et al. (2000) were performed using simple assumptions about the dynamo and a more realistic modeling is needed. However, the results are already very promising (see Ferreira et al. 2000 for more details).

It was found that all low-mass Class 0 objects can indeed be spun down, from the break-up speed to about $10 \%$ of it, on a time scale consistent with the duration of the embedded phase for very reasonable values of the free parameters $(n=3$ or $4, f \lambda>0.1)$. Stellar period, mass, radius and disc accretion rates were found consistent with values for $\mathrm{T}$ Tauri stars with a dipole field smaller than $1 \mathrm{kG}$. Finally, it is noteworthy that the main difference between "accretion-powered stellar winds" and Reconnection X-winds relies on the stellar magnetic moment. In the former case, it is anti-parallel to the disc field while it is parallel in the latter. If the dynamo action explicitly assumed provides a magnetic field reversal, then recurrent transitions from one wind configuration to another can be expected.

\section{Conclusion}

The theory of steady jet production from Keplerian accretion discs has been completed in the framework of "alpha" discs. The physical conditions required to thermomagnetically drive jets are constrained and all the relevant physical processes have been included. The role of large scale magnetic fields in discs has gradually emerged and it seems now an unavoidable ingredient of star formation theory as a whole. The progresses in star-disc interaction reported here provide valuable insights but one should remain cautious as stellar magnetic fields are not pure, aligned dipoles.

The amount of magnetic flux $\Phi$ in the disc is an unknown parameter but it is reasonable to assume that it scales with the total mass $M$. If this is verified then two important aspects could be naturally explained:

(1) Reconnection $\mathrm{X}$-winds can brake down a protostar during the embedded phase, explaining that $\mathrm{T}$ Tauri stars rotate at about $10 \%$ of the break-up speed. Remarkably, the mystery of the low dispersion in angular velocities would be naturally accounted by a low dispersion in the ratio $\Phi / M$ (Ferreira et al. 2000). These winds are also a very promising means to drive time dependent massive bullets, channeled by the outer steady disc wind. 
(2) The transition from disc winds (Class 0, I, II) to stellar winds (Class II, III) would follow the evolution of the disc magnetic flux $\Phi$, with a transition radius between the outer SAD and the inner JED decreasing in time.

\section{References}

Armitage, P. J. \& Clarke, C. J. 1996, MNRAS, 280, 458

Balbus, S. A. 2003, ARAA, 41, 555

Banerjee, R. \& Pudritz, R. E. 2006, ApJ, 641, 949

Basu, S. \& Mouschovias, T. C. 1994, ApJ, 432, 720

Blandford, R. D. \& Payne, D. G. 1982, MNRAS, 199, 883

Bouvier, J., Alencar, S. H. P., Harries, T. J., Johns-Krull, C. M., \& Romanova, M. M. 2007, in Protostars and Planets V, ed. B. Reipurth, D. Jewitt, \& K. Keil, 479-494

Bouvier, J., Wichmann, R., Grankin, K., et al. 1997, A\&A, 318, 495

Cabrit, S., Edwards, S., Strom, S. E., \& Strom, K. M. 1990, ApJ, 354, 687

Casse, F. \& Ferreira, J. 2000, A\&A, 353, 1115

Casse, F. \& Keppens, R. 2002, ApJ, 581, 988

Casse, F. \& Keppens, R. 2004, ApJ, 601, 90

Collier Cameron, A. \& Campbell, C. G. 1993, A\&A, 274, 309

DeCampli, W. M. 1981, ApJ, 244, 124

Donati, J.-F., Paletou, F., Bouvier, J., \& Ferreira, J. 2005, Nature, 438, 466

Ferreira, J. 1997, A\&A, 319, 340

Ferreira, J. \& Casse, F. 2004, ApJ1, 601, L139

Ferreira, J., Dougados, C., \& Cabrit, S. 2006a, $A \& A$, in press

Ferreira, J. \& Pelletier, G. 1995, A\&A, 295, 807

Ferreira, J., Pelletier, G., \& Appl, S. 2000, MNRAS, 312, 387

Ferreira, J., Petrucci, P.-O., Henri, G., Saugé, L., \& Pelletier, G. 2006b, A\&AA, 447, 813

Ghosh, P., Pethick, C. J., \& Lamb, F. K. 1977, ApJ, 217, 578

Glassgold, A. E., Najita, J., \& Igea, J. 2004, ApJ, 615, 972

Hartigan, P., Edwards, S., \& Ghandour, L. 1995, ApJ, 452, 736

Hartmann, L. \& MacGregor, K. B. 1980, ApJ, 242, 260

Heiles, C., Goodman, A. A., McKee, C. F., \& Zweibel, E. G. 1993, in Protostars and Planets III, ed. E. H. Levy \& J. I. Lunine, 279-326

Hirose, S., Uchida, Y., Shibata, K., \& Matsumoto, R. 1997, PASJ, 49, 193

Konigl, A. 1989, ApJ, 342, 208

Kwan, J. 1997, ApJ, 489, 284

Long, M., Romanova, M. M., \& Lovelace, R. V. E. 2005, ApJ, 634, 1214

Lovelace, R. V. E., Li, H., Colgate, S. A., \& Nelson, A. F. 1999, ApJ, 513, 805

Lovelace, R. V. E., Romanova, M. M., \& Bisnovatyi-Kogan, G. S. 1995, MNRAS, 275, 244

Machida, M. N., Inutsuka, S.-i., \& Matsumoto, T. 2006, ApJl, 647, L151

Masset, F. S., Morbidelli, A., Crida, A., \& Ferreira, J. 2006, ApJ, 642, 478

Matt, S. \& Pudritz, R. E. 2005a, ApJl, 632, L135

Matt, S. \& Pudritz, R. E. 2005b, MNRAS, 356, 167

Ménard, F. \& Duchêne, G. 2004, A\& $A, 425,973$

Miller, K. A. \& Stone, J. M. 2000, ApJ, 534, 398

Moss, D. 2004, A\&A, 414, 1065

Najita, J. R., Carr, J. S., Glassgold, A. E., \& Valenti, J. 2007, ArXiv e-prints, 704

Pringle, J. E. \& Rees, M. J. 1972, A\& A, 21, 1

Rebull, L. M., Wolff, S. C., Strom, S. E., \& Makidon, R. B. 2002, AJ, 124, 546

Shakura, N. I. \& Sunyaev, R. A. 1973, A\& A, 24, 337

Stone, J. M., Hawley, J. F., Gammie, C. F., \& Balbus, S. A. 1996, ApJ, 463, 656

Strom, K. M., Strom, S. E., Wolff, S. C., Morgan, J., \& Wenz, M. 1986, ApJs, 62, 39

Uchida, Y. \& Low, B. C. 1981, Journal of Astrophysics and Astronomy, 2, 405

Zanni, C., Ferrari, A., Rosner, R., Bodo, G., \& Massaglia, S. 2007, A\&AA, 469, 811 